\title{
Perturbative tests of non-perturbative counting
}

\author{
Atish Dabholkar ${ }^{a, b}$ and João Gomes ${ }^{b}$ \\ ${ }^{a}$ Laboratoire de Physique Théorique et Hautes Energies (LPTHE), \\ Université Pierre et Marie Curie-Paris 6; CNRS UMR 7589, \\ Tour 24-25, $5^{e ̀ m e}$ étage, Boite 126, 4 Place Jussieu, 75252 Paris Cedex 05, France \\ ${ }^{b}$ Department of Theoretical Physics, Tata Institute of Fundamental Research, \\ Homi Bhabha Rd, Mumbai 400 005, India \\ E-mail: atish@lpthe.jussieu.fr, gomes@lpthe.jussieu.fr
}

Abstract: We observe that a class of quarter-BPS dyons in $\mathcal{N}=4$ theories with charge vector $(Q, P)$ and with nontrivial values of the arithmetic duality invariant $I:=\operatorname{gcd}(Q \wedge P)$ are nonperturbative in one frame but perturbative in another frame. This observation suggests a test of the recently computed nonperturbative partition functions for dyons with nontrivial values of the arithmetic invariant. For all values of $I$, we show that the nonperturbative counting yields vanishing indexed degeneracy for this class of states everywhere in the moduli space in precise agreement with the perturbative result.

Keywords: Black Holes in String Theory, Superstrings and Heterotic Strings, String Duality

ArXiv ePrint: 0911.0586 


\section{Contents}

1 Introduction $\quad 1$

2 A class of states 3

3 Nonperturbative counting $\quad 4$

4 Perturbative counting $\quad 4$

$\begin{array}{lll}5 & \text { A test } & 7\end{array}$

\section{Introduction}

Quarter-BPS dyons in string compactifications with $\mathcal{N}=4$ supersymmetry in four dimensions offer a particularly tractable framework for understanding exact quantum properties of black holes. In some models, it has recently become possible to compute the exact indexed degeneracies of all duality orbits of these dyons at all points in the moduli space. The spectrum reveals an intricate moduli dependence and a complicated structure of walls of marginal stability which is nevertheless precisely computable. Such detailed knowledge of the microscopic spectrum has made it possible now to probe some of the finer aspects of black hole physics such as subleading corrections to the quantum Wald entropy, both perturbative $[1,2]$ and even nonperturbative [3-5].

In this note we consider some simple but nontrivial perturbative tests of these nonperturbative counting formulae for quarter-BPS dyons. We consider, for purposes of illustration, the simplest model with $\mathcal{N}=4$ supersymmetry obtained by compactifying the heterotic string on $T^{6}$. The U-duality group $G(\mathbb{Z})$ in this case is

$$
\mathrm{SL}(2, \mathbb{Z}) \times O(6,22 ; \mathbb{Z})
$$

In the heterotic frame, the first factor corresponds to the electric-magnetic duality and the second factor corresponds to T-duality. A dyon with an electric charge vector $Q^{i}$ and magnetic charge vector $P^{i}$ has charge vector

$$
\Gamma=\left[\begin{array}{l}
Q^{i} \\
P^{i}
\end{array}\right],
$$

where the index $i$ transforms in the vector representation of $O(6,22 ; \mathbb{Z})$ and the doublet $(Q, P)$ transforms in the fundamental representation of $\mathrm{SL}(2, \mathbb{Z})$. If the vectors $Q$ and $P$ are parallel then the BPS state preserves one half of the supersymmetries, if not only a quarter of the supersymmetries. 
A major simplification in this model results from the fact that all inequivalent duality orbits can be completely classified. ${ }^{1}$ For the purposes of dyon counting, inequivalent duality orbits are labeled essentially by a single integer $I$ which is a duality invariant [6-8] defined by

$$
I=\operatorname{gcd}(Q \wedge P) .
$$

We refer to $I$ as an arithmetic duality invariant to underscore the fact that it is an invariant of the arithmetic duality group $G(\mathbb{Z})$ which cannot be expressed as an invariant of the continuous duality group $G(\mathbb{R})$. For each $I$, one can define the matrix of T-duality invariants

$$
\Lambda=\left(\begin{array}{cc}
Q^{2} & Q \cdot P \\
Q \cdot P & P^{2}
\end{array}\right)=\left(\begin{array}{cc}
2 n & l \\
l & 2 m
\end{array}\right) .
$$

Integrality of $(n, m, l)$ follows from the fact that the charge lattice is even integral. The counting is then captured by a partition function $Z_{I}(\Omega)$ that depends on the matrix

$$
\Omega=\left(\begin{array}{cc}
\tau & z \\
z & \sigma
\end{array}\right)
$$

of the three chemical potentials $(\tau, \sigma, z)$ conjugate to the integers $(n, m, l)$ respectively. $^{2}$ The dyon degeneracies for a given value of $I$ are given by Fourier coefficients of of $Z_{I}(\Omega)$. The degeneracies are moduli dependent because the Fourier coefficients depend on the choice of the Fourier contours which in turn depend on the moduli in a precise way $[6,9,10]$.

These counting formulae, which we review in $\S 3$, were obtained generalizing earlier work for $I=1[2,11-15]$. Partition functions for arbitrary values $I$ were proposed first from macroscopic considerations $[16,17]$ so that the Wald entropy of corresponding black holes and the structure of wall-crossings is correctly reproduced. A microscopic two-dimensional superconformal field theory was proposed for this system in [18] from considerations of instanton moduli space in multi KK-monopole background.

Since there are several subtleties associated with the instanton moduli space and especially the moduli space of multi KK-monopoles, it is desirable to have additional tests of these counting formulae, which are at the same time independent of considerations of Wald entropy and wall crossings. One such useful microscopic test was devised in [19, 20] by considering a class of charge configurations that can be realized both in string theory and in nonabelian gauge theory. Comparing with the field theory counting obtained using very different methods, one obtains a successful test of the stringy counting. Moreover, since these states have very small charges, the test is independent of considerations from black hole physics which corresponds to the opposite limit of large charges.

\footnotetext{
${ }^{1}$ We note in passing that a classification of duality orbits is more subtle for models with both higher and lower supersymmetry. For example, a complete classification of orbits of the $E_{7,7}(\mathbb{Z})$ duality group for $\mathcal{N}=8$ is not yet known. In models with $\mathcal{N}=2$ supersymmetry, on the other hand, the moduli space typically receives large quantum corrections and the precise form of the arithmetic duality group itself is often not known.

${ }^{2}$ More accurately, one has a collection of partition functions that can be obtained from $Z_{I}$ by the action of an element of $\operatorname{SL}(2, \mathbb{Z}) / \Gamma^{0}(I)$ that is determined by arithmetic T-duality invariants [8].
} 
In this note we devise another independent microscopic test the string theory counting formulae. Our strategy will be to identify some states that are nonperturbative in one frame but are perturbative in another. A similar strategy has of course been used very successfully for half-BPS states in the study of various dualities. However, in $\mathcal{N}=4$ gauge theory, the quarter-BPS states are necessarily nonperturbative and can never be mapped to any perturbative states. This is because the only perturbative BPS states in gauge theory are the gauge bosons which are half-BPS. This might lead one to expect that the same is true also in string theory. Amusingly, this is not the case and some quarter-BPS states in string theory do map to perturbative states. Moreover, these states, even though perturbative, have nontrivial values of the arithmetic invariant. Consequently, one obtains a simple test of the microscopic counting formulae for quarter-BPS dyons for nontrivial values of $I$ by comparing it with the perturbative counting.

\section{A class of states}

Without loss of generality one can work at a point in the moduli space where the $T^{6}$ is a product $T^{4} \times T^{2}$ because the partition functions are independent of moduli. One can further focus on a smaller charge sector invariant under $\mathrm{SO}(2,2) \subset \mathrm{SO}(6,22)$ using U-duality. In this sector, the dyon has the following charge configuration

$$
\Gamma=\left[\begin{array}{l}
Q \\
P
\end{array}\right]=\left[\begin{array}{ccc}
\tilde{n}, & n ; & \tilde{w}, w \\
\tilde{W}, & W ; & \tilde{K}, K
\end{array}\right]
$$

In the heterotic frame, the gauge fields associated with these charges arise from the reduction of the metric and the antisymmetric B field along a $T^{2} \sim S \times \tilde{S}$. The charges $n$ and $w$ represent, respectively, the momenta and winding along the circle $S$. The $K$ charge corresponds to Kaluza-Klein monopole associated with the circle $S$, and $W$ represents the charge of NS5-branes associated with the circle $S$ but wrapping $T^{4} \times \tilde{S}$. The charges with the tilde are the analogues for the $\tilde{S}$ circle.

The charge configuration of our interest is of the following form:

$$
\Gamma_{n}=\left[\begin{array}{l}
Q \\
P
\end{array}\right]=\left[\begin{array}{l}
0, n ; 0,0 \\
1,0 ; 0,0
\end{array}\right] .
$$

It is evident that the matrix of duality invariants $\Lambda$ defined in (1.4) vanishes for these states but the arithmetic duality invariant $I$ defined in (1.3) is nevertheless nontrivial and equals $n$. Moreover, since the electric charge vector is not parallel to the magnetic charge vector the state is quarter-BPS and not half-BPS.

Under six-dimensional string-string duality, the heterotic NS5-brane is mapped to Type-IIA fundamental string, and the momenta are mapped to momenta. Thus, in the Type-II frame, our state corresponds to a perturbative Type-II fundamental string with winding number one with $n$ units of momentum along the $S$ circle. We now proceed to discuss the nonperturbative and perturbative counting of these states. 


\section{$3 \quad$ Nonperturbative counting}

Let us summarize the prescription for extracting the nonperturbative degeneracies. At a given point $\mu$ in the moduli space, the degeneracies for $I=1$, are given by the fourier coefficients

$$
\left.d_{1}(\Lambda)\right|_{\mu}=\int_{\mathcal{C}(\mu)} d \Omega \frac{e^{-\pi i \operatorname{Tr}(\Omega \Lambda)}}{\Phi_{10}(\Omega)} .
$$

where $\Phi_{10}$ is the well-known Igusa cusp form that transforms as Siegel modular form of $\operatorname{Sp}(2, \mathbb{Z})$ with weight ten [11]. The precise dependence of the contour $\mathcal{C}(\mu)$ on the moduli is as in [10].

For general values of $I$, we first choose a charge configuration of the form

$$
\Gamma=\left[\begin{array}{c}
Q=I Q_{0} \\
P_{0}
\end{array}\right]
$$

with $\operatorname{gcd}(Q \wedge P)=I$. The degeneracies $d_{I}$ are then given by the Fourier coefficients of a modified elliptic genus of this superconformal field theory [18], and can be expressed in terms of $d_{1}$ :

$$
\left.d_{I}(\Lambda)\right|_{\mu}=\left.\sum_{s \mid I} s d_{1}\left(\Lambda_{s}\right)\right|_{\mu},
$$

where we have defined

$$
\Lambda_{s}=\left(\begin{array}{cc}
Q^{2} / s^{2} & Q \cdot P / s \\
Q \cdot P / s & P^{2}
\end{array}\right) .
$$

Note that $\Lambda_{s}$ thus defined has integral entries for the charge configuration (3.2). More general charge configurations with same values of $I$ but different values of the arithmetic T-duality invariants can be first brought to this form by an S-duality transformation [8] which is an element of $\operatorname{SL}(2, \mathbb{Z}) / \Gamma^{0}(I)$. Then the degeneracies are defined as above to ensure S-duality invariance.

The degeneracy defined by the formulae (3.1) and (3.3) evidently has a complicated dependence on moduli. We will find though in $\S 5$ that for our states which have $\Lambda=0$, the moduli dependence disappears. Moreover, the degeneracy in fact vanishes because this particular Fourier coefficient of $1 / \Phi_{10}$ is zero. Both these facts agree with the perturbative counting which we now describe.

\section{Perturbative counting}

Our charge configuration (2.2) maps to a perturbative state in IIA compactified on $K 3 \times$ $S^{1} \times \tilde{S}^{1}$ fundamental string with unit winding and $n$ units of momentum along $S^{1}$. The computation of the perturbative degeneracies is straightforward but not entirely trivial. In particular, we will see that the indexed degeneracy does not vanish for half-BPS states but does vanish for quarter-BPS states.

For this purpose, we choose the light-cone gauge in Green-Schwarz formalism, and we work in the orbifold limit of $K 3 \sim T^{4} / \mathbb{Z}_{2}$. The worldsheet field thus have a target manifold 
$\mathbb{R}^{2} \times T^{2} \times T^{4} / \mathbb{Z}_{2}$. The fields are classified according to $\operatorname{Spin}(2)_{1} \times \operatorname{Spin}(2)_{2} \times \mathrm{SU}(2)_{L} \times \mathrm{SU}(2)_{R}$ representations where the first two $\operatorname{Spin}(2)$ factors are the tangent space rotations in $\mathbb{R}^{2}$ and $T^{2}$ whereas the last two $\mathrm{SU}(2)$ factors are the tangent space rotations of the un-orbifolded $T^{4}$. The light-cone Green-Schwarz fermions transform as

$$
\begin{aligned}
& 8_{s}=\left(+\frac{1}{2} ;+\frac{1}{2} ; 2,1\right) \oplus\left(+\frac{1}{2} ;-\frac{1}{2} ; 1,2\right) \oplus\left(-\frac{1}{2} ;+\frac{1}{2} ; 1,2\right) \oplus\left(-\frac{1}{2} ;-\frac{1}{2} ; 2,1\right) \\
& 8_{c}=\left(+\frac{1}{2} ;-\frac{1}{2} ; 2,1\right) \oplus\left(+\frac{1}{2} ;+\frac{1}{2} ; 1,2\right) \oplus\left(-\frac{1}{2} ;-\frac{1}{2} ; 1,2\right) \oplus\left(-\frac{1}{2} ;+\frac{1}{2} ; 2,1\right)
\end{aligned}
$$

The orbifold $\mathbb{Z}_{2}$ acts on the $\mathrm{SU}(2)_{R}$ factor. Consequently the representation $(1,2)$ is projected out and we are left with eight real fermion zero modes. The eight bosons transform as

$$
8_{v}=( \pm 1 ; 0 ; 1,1) \oplus(0 ; \pm 1 ; 1,1) \oplus(0 ; 0 ; 2,2) .
$$

We want to compute the partition function

$$
Z(q, \bar{q}, y)=\operatorname{Tr}(-1)^{F_{L}+F_{R}} q^{L_{0}} \bar{q}^{\bar{L}_{0}} y^{2 J},
$$

where the trace is taken over oscillator modes. The $J$ operator is the generator of the $\operatorname{Spin}(2)_{1}$. Tracing over the oscillator states gives

$$
\begin{gathered}
Z(q, \bar{q}, y)=\left(y^{\frac{1}{2}}-y^{-\frac{1}{2}}\right)^{4} \prod_{n \geq 1, j= \pm 1} \frac{\left(1-\bar{q}^{n} y^{j}\right)^{2}\left(1-q^{n} y^{j}\right)^{2}}{\left(1-\bar{q}^{n}\right)^{2}\left(1-\bar{q}^{n} y^{2 j}\right)\left(1-q^{n}\right)^{2}\left(1-q^{n} y^{2 j}\right)} \times \\
\times \operatorname{Tr}_{K 3}(-1)^{F_{L}+F_{R}} q^{L_{0}} \bar{q}^{\bar{L}_{0}} y^{2 J} .
\end{gathered}
$$

The last $K 3$ factor corresponds to the fields which transform under $\mathrm{SU}(2)_{R}$ and get twisted. There are four bosons that transform as $(0 ; 0 ; 2,2)$ and fermions that transform as

$$
\begin{array}{cl}
\text { left-moving: } & \left(+\frac{1}{2} ;-\frac{1}{2} ; 1,2\right) \oplus\left(-\frac{1}{2} ;+\frac{1}{2} ; 1,2\right) \\
\text { right-moving: } & \left(+\frac{1}{2} ;+\frac{1}{2} ; 1,2\right) \oplus\left(-\frac{1}{2} ;-\frac{1}{2} ; 1,2\right) .
\end{array}
$$

Tracing over oscilator modes of these fields gives

$$
\begin{aligned}
\operatorname{Tr}_{K 3}(-1)^{F_{L}+F_{R}} q^{L_{0}} \bar{q}^{\bar{L}_{0}} y^{2 J}= & 8\left[\frac{\vartheta_{2}(\tau, \nu)^{2} \vartheta_{2}(\bar{\tau}, \nu)^{2}}{\vartheta_{2}(\tau, 0)^{2} \vartheta_{2}(\bar{\tau}, 0)^{2}}+\frac{\vartheta_{3}(\tau, \nu)^{2} \vartheta_{3}(\bar{\tau}, \nu)^{2}}{\vartheta_{3}(\tau, 0)^{2} \vartheta_{3}(\bar{\tau}, 0)^{2}}+\frac{\vartheta_{4}(\tau, \nu)^{2} \vartheta_{4}(\bar{\tau}, \nu)^{2}}{\vartheta_{4}(\tau, 0)^{2} \vartheta_{4}(\bar{\tau}, 0)^{2}}\right] \\
& +\left(y^{\frac{1}{2}}-y^{-\frac{1}{2}}\right)^{4}(\ldots)
\end{aligned}
$$

The last term, which we denote by dots, will not be important for our computation as it contributes with additional fermion zero modes.

The quarter-BPS dyons break twelve supersymmetries which lead to six complex fermion zero modes. Hence the degeneracy is captured by a helicity supertrace $B_{6}$ which can extracted from $Z(q, \bar{q}, y)$ by acting with six $y$ derivatives before setting $y=1[21,22]$. This particular helicity supertrace has been computed in [23] and found to be zero as a 
result of an accidental cancelation between quarter-BPS multiplets which is not a consequence of supersymmetry. We present the computation in a slightly different form below.

Note that $Z(q, \bar{q}, y)$ has explicitly a factor of $\left(y^{1 / 2}-y^{-1 / 2}\right)^{4}$, which means that we only need to take two further derivatives on the $(q, \bar{q})$ dependent piece. That is

$$
\begin{aligned}
&\left.\frac{1}{2} \frac{d^{2}}{d y^{2}}\left[\frac{Z(q, \bar{q}, y)}{\left(y^{1 / 2}-y^{-1 / 2}\right)^{4}}\right]\right|_{y=1} \\
&=\frac{1}{2} \frac{d^{2}}{d y^{2}}\left\{\prod_{n \geq 1, j= \pm 1} \frac{\left(1-\bar{q}^{n} y^{j}\right)^{2}\left(1-q^{n} y^{j}\right)^{2}}{\left(1-\bar{q}^{n}\right)^{2}\left(1-\bar{q}^{n} y^{2 j}\right)\left(1-q^{n}\right)^{2}\left(1-q^{n} y^{2 j}\right)}\right\}_{y=1} \times 24 \\
& \quad+\frac{1}{2} \frac{d^{2}}{d y^{2}}\left\{\operatorname{Tr}_{K 3}(-1)^{F_{L}+F_{R}} q^{L_{0}} \bar{q}^{\bar{L}_{0}} y^{2 J}\right\}_{y=1} .
\end{aligned}
$$

Because the partition function is $q \longleftrightarrow \bar{q}$ symmetric we will have quarter-BPS states by exciting either the left or right-moving sectors. We consider the states with right-movers in the ground states with arbitrary left-moving oscillations with degeneracy $d(m)$. The generating function for these degeneracies is

$$
\begin{aligned}
\sum_{m} d(m) q^{m} & =16 \times\left[\sum_{s \geq 1} \sum_{n \geq 1} s\left(3-(-1)^{s}\right) q^{n s}-s\left(1+(-1)^{s}\right) q^{\left(n-\frac{1}{2}\right) s}\right] \\
& =16 \times \sum_{s \geq 1} \sum_{n \geq 1} s\left(3-(-1)^{s}\right) q^{n s}-\sum_{s \geq 1} \sum_{n \geq 1} 64 s q^{\left(n-\frac{1}{2}\right) 2 s} .
\end{aligned}
$$

The level matching condition is

$$
L_{0}-\bar{L}_{0}=n w=I
$$

where $w$ and $n$ are respectively the winding and momenta along the circle $S$. The BPS condition sets $\bar{L}_{0}=0$. Setting $L_{0}=I$ for our configuration yields

$$
d(I)=16\left[\sum_{s \mid I} s\left(3+(-1)^{s+1}\right)-4 \sum_{(2 s+1) \mid I} \frac{I}{2 s+1}\right]
$$

This strange sum over divisors actually vanishes. To see this note that any number $I$ can be written as $I=2^{N} I_{\text {odd }}$, for some $N$, where $I_{\text {odd }}$ is odd. The complete set of divisors of $I$ is

$$
\left\{2^{i} \hat{s}_{j}\right\}
$$

where $i$ goes from 0 to $N$ and $j$ runs through the divisors of $I_{\text {odd }}$. The sum (4.10) simplifies to

$$
\begin{aligned}
d(I) & =16\left[\sum_{\hat{s}} 4 \hat{s}+2 \hat{s} \sum_{i=1}^{N} 2^{i}-4.2^{N} \hat{s}\right] \\
& =16\left[\sum_{\hat{s}} 4 \hat{s}+4 \hat{s}\left(2^{N}-1\right)-4.2^{N} \hat{s}\right]=0
\end{aligned}
$$


The same result was found in [23] as a consequence a theta identity eq. (B.22).

Note that for $n=0$, we actually have a half-BPS state which is dual the perturbative state also of the heterotic string. Since it breaks only eight supersymmetries, there are only four complex fermion zero modes. Hence we need to take only the fourth derivative of the partition function (4.5) to compute the helicity supertrace $B_{4}$. For $B_{4}$, one correctly obtains a nonzero multiplicity which moreover equals 24 consistent with the heterotic counting [21]. We thus see that vanishing of $B_{6}$ in our case is accidental. It is not a consequence of cancelation between bosons and fermions within a multiplet but rather of cancelation between full supermultiplets.

\section{A test}

We would now like to reproduce two interesting facts about the perturbative counting from the perspective of the nonperturbative counting.

First, the degeneracy of perturbative states is expected to be moduli independent. On the other hand, the nonperturbative spectrum a priori has a sensitive moduli dependence. The states could even decay upon crossing walls of marginal stability. For the class of states, that we have considered, however, the moduli dependence disappears. To see this, we note first that $d_{1}$ is S-duality covariant

$$
\left.d_{1}\left(\Lambda^{\prime}\right)\right|_{\mu^{\prime}}=\left.d_{1}(\Lambda)\right|_{\mu}
$$

and thus the degeneracy of a given configuration $\Lambda$ at a given point in the moduli space $\mu$ equals the degeneracy of the dual charge configuration $\Lambda^{\prime}$ at another point $\mu^{\prime}$ in the moduli space which is an image of $\mu$ under S-duality. Now since $\Lambda=0$ is invariant under with S-duality, we have

$$
\left.d_{1}(0)\right|_{\mu^{\prime}}=\left.d_{1}(0)\right|_{\mu}
$$

Furthermore, using the natural embedding of the $\operatorname{SL}(2, \mathbb{Z})$ S-duality group in $\operatorname{Sp}(2, \mathbb{Z})$, it is known that moduli space is divided into chambers separated by walls and one can go from any chamber to any other chamber by an S-duality transformation. One can thus cover the entire moduli space by S-duality transformations starting from any given chamber [10]. Therefore, using (3.3) we conclude that $\left.d_{I}(0)\right|_{\mu}$ is independent of $\mu$ for any $\mu$.

Second, it is easy to check from the expansion of the explicit expression for $1 / \Phi_{10}$ that $d_{1}(0)=0$ and hence we conclude from (3.3) that

$$
d_{I}(0)=0 .
$$

Note that the vanishing of $d_{1}(0)$ itself is a consequence of a peculiar fact about $1 / \Phi_{10}$ that a particular Fourier coefficient vanishes which is not true for a general Siegel form. The vanishing of $d_{I}(0)$ depends in addition on the fact that $d_{I}$ is expressible in terms of $d_{1}$ as in (3.3). Thus, the vanishing of degeneracies for this specific charge configurations, even though simple to verify, constitutes a nontrivial test of (3.3). Recall that our degeneracies are actually indexed degeneracies and hence they can be zero or even negative.

In conclusion, both the actual degeneracy and the moduli dependence of the nonperturbative counting is in complete agreement with the perturbative counting. 


\section{Acknowledgments}

It is a pleasure to thank Sameer Murthy and Suresh Nampuri for early collaboration. We would like to thank Boris Pioline and Ashoke Sen for comments on the draft. The work of A. D. was supported in part by the Excellence Chair of the Agence Nationale de la Recherche (ANR). The work of J.G. was supported in part by Fundação para a Ciência e Tecnologia (FCT).

Open Access. This article is distributed under the terms of the Creative Commons Attribution Noncommercial License which permits any noncommercial use, distribution, and reproduction in any medium, provided the original author(s) and source are credited.

\section{References}

[1] G. Lopes Cardoso, B. de Wit, J. Kappeli and T. Mohaupt, Asymptotic degeneracy of dyonic $N=4$ string states and black hole entropy, JHEP 12 (2004) 075 [hep-th/0412287] [SPIRES].

[2] J.R. David and A. Sen, CHL dyons and statistical entropy function from D1 - D5 system, JHEP 11 (2006) 072 [hep-th/0605210] [SPIRES].

[3] N. Banerjee, D.P. Jatkar and A. Sen, Asymptotic expansion of the $N=4$ dyon degeneracy, JHEP 05 (2009) 121 [arXiv:0810.3472] [SPIRES].

[4] A. Sen, Arithmetic of quantum entropy function, JHEP 08 (2009) 068 [arXiv:0903.1477] [SPIRES].

[5] S. Murthy and B. Pioline, A Farey tale for $N=4$ dyons, JHEP 09 (2009) 022 [arXiv: 0904 .4253] [SPIRES].

[6] A. Dabholkar, D. Gaiotto and S. Nampuri, Comments on the spectrum of CHL dyons, JHEP 01 (2008) 023 [hep-th/0702150] [SPIRES].

[7] S. Banerjee and A. Sen, Duality orbits, dyon spectrum and gauge theory limit of heterotic string theory on $T^{6}$, JHEP 03 (2008) 022 [arXiv: 0712.0043] [SPIRES].

[8] S. Banerjee and A. Sen, S-duality action on discrete T-duality invariants, JHEP 04 (2008) 012 [arXiv:0801.0149] [SPIRES].

[9] A. Sen, Walls of marginal stability and dyon spectrum in $N=4$ supersymmetric string theories, JHEP 05 (2007) 039 [hep-th/0702141] [SPIRES].

[10] M.C.N. Cheng and E. Verlinde, Dying dyons don't count, JHEP 09 (2007) 070 [arXiv:0706.2363] [SPIRES].

[11] R. Dijkgraaf, E.P. Verlinde and H.L. Verlinde, Counting dyons in $N=4$ string theory, Nucl. Phys. B 484 (1997) 543 [hep-th/9607026] [SPIRES].

[12] D. Gaiotto, A. Strominger and X. Yin, New connections between $4 D$ and $5 D$ black holes, JHEP 02 (2006) 024 [hep-th/0503217] [SPIRES].

[13] D. Gaiotto, Re-recounting dyons in $N=4$ string theory, hep-th/0506249 [SPIRES].

[14] D. Shih, A. Strominger and X. Yin, Recounting dyons in $N=4$ string theory, JHEP 10 (2006) 087 [hep-th/0505094] [SPIRES]. 
[15] D. Shih and X. Yin, Exact black hole degeneracies and the topological string, JHEP 04 (2006) 034 [hep-th/0508174] [SPIRES].

[16] S. Banerjee, A. Sen and Y.K. Srivastava, Partition functions of torsion $>1$ dyons in heterotic string theory on $T^{6}$, JHEP 05 (2008) 098 [arXiv: 0802.1556] [SPIRES].

[17] S. Banerjee, A. Sen and Y.K. Srivastava, Generalities of quarter BPS dyon partition function and dyons of torsion two, JHEP 05 (2008) 101 [arXiv:0802.0544] [SPIRES].

[18] A. Dabholkar, J. Gomes and S. Murthy, Counting all dyons in $N=4$ string theory, arXiv:0803.2692 [SPIRES].

[19] A. Sen, Three string junction and $N=4$ dyon spectrum, JHEP 12 (2007) 019 [arXiv: 0708.3715] [SPIRES].

[20] A. Dabholkar, K. Narayan and S. Nampuri, Degeneracy of decadent dyons, JHEP 03 (2008) 026 [arXiv:0802.0761] [SPIRES].

[21] E. Kiritsis, Introduction to superstring theory, hep-th/9709062 [SPIRES].

[22] E. Kiritsis, Introduction to non-perturbative string theory, hep-th/9708130 [SPIRES].

[23] A. Gregori et al., $R^{2}$ corrections and non-perturbative dualities of $N=4$ string ground states, Nucl. Phys. B 510 (1998) 423 [hep-th/9708062] [SPIRES]. 